\title{
Novel Wnt and Notch Signaling Natural Inhibitors as Double Edged Sword Against Cancer War: An approach Towards Computer Based Drug Design
}

\author{
Shashank Kumar* \\ Centre for Biochemistry and Microbial Sciences, Central University of Punjab, India
}

Submission: May 15, 2016; Published: May 25, 2016

*Corresponding author: Shashank Kumar, Assistant Professor, Centre for Biochemistry and Microbial Sciences, Central University of Punjab, Bathinda, India, 151001, Tel: +91 9335647413; Email: shashankbiochemau@gmail.com

\begin{abstract}
Editorial
Cancer is a group of disease characterized by the uncontrolled growth and spread of abnormal cells which may lead to death. According to a report by GLOBOCAN the number of new cancer cases would increase to 19.3 million by 2025 worldwide. Thus globally cancer has become a big threat to human beings, among various diseases. Carcinogen induced deregulation in cellular signaling pathway, may leads to the cancer development. Chemotherapy is an important mode of cancer treatment that used to cure and improve the patient's quality of life [1]. At the same time chemotherapy is known to induce drug resistance and toxicity that hurdles the improvement of overall response and survival of cancer patients. Many cancers contain a sub-population of self-renewing and expanding stem cells known as cancer stem cells (CSCs). These are associated with chemotherapeutic resistant leading to tumor recurrence and poor patient prognosis. Acquired drug resistance renders subsequent anticancer therapy effectiveness leading to tumor recurrence and progression. Hence it is urgency of time to identify effective and safer anticancer agents, which can be found in natural agents. The use of natural products is promising because they target multiple signaling pathways and have minimal toxicity compared to conventional chemotherapeutics. Malignancy transformation and progression are multistage progress involved gene alterations and multi signaling pathways. In this regard herbal products may do a lot. Several natural compounds viz., vinblastine, etoposide, paclitaxel, camptothecin, bleomycin, and doxorubicin are known to prevent the occurrence and/or spread of various cancers by targeting numerous key elements in intracellular signaling network involved in carcinogenesis and is being investigated in clinical
\end{abstract} trials.

Abbreviations: CSCs: Cancer Stem Cells; NICD: Notch Intracellular Domain; TACE: Tumor Necrosis Factor- $\alpha$-Converting Enzyme; CADD: Computer Aided Drug Designing; ADME $\backslash$ T: Absorption, Distribution, Metabolism, Excretion and Toxicity

\section{Introduction}

Different signaling pathways play critical role in maintaining the homeostasis of cell proliferation, differentiation and apoptosis. Wnt and Notch signaling pathways have been reported to play role in various kind of cancer. Wht signaling is involved in cell proliferation, cell migration and developmental processes. On the other hand Notch pathway beside developmental process has been well documented for its role in cancer stem cells survival (self renewal ability) and drug resistance. The binding of the Wnt proteins to the Frizzled receptor allows $\beta$-catenin, the key mediator of Wnt signaling, to accumulate in the cytoplasm and then translocate into the nucleus. In the nucleus, it regulates target gene expressions which are involved in homeostasis of cell growth and other cellular functions as well. Notch signaling has been reported to maintain the cancer stem-cell (CSCs) pool by the process of self-renewal, epithelial-mesenchymal transition (metastasis) induction and chemo-resistance [2]. CSCs are associated with chemotherapeutic resistant and tumor recurrence leading to poor prognosis. Binding of Notch ligand and its receptor, between two neighboring cells activates Notch signaling. Upon activation the cleaved Notch releases Notch intracellular domain (NICD) through a cascade of metalloprotease mediated proteolytic cleavage, tumor necrosis factor- $\alpha$-converting enzyme (TACE) and $\gamma$-secretase. NICD translocate to nucleus and transcriptionally activates Notch target genes. Inhibition of $\gamma$-secretase function may prevent the cleavage of the Notch receptor and blocks Notch signal transduction. Currently, several classes of Notch inhibitors have been developed but $\gamma$-secretase inhibitors are the most common studied tool for Notch signaling inhibition. Several tumor types often contain over activated $\mathrm{Wnt} / \beta$-catenin and/or 
Notch signaling cascade. Some proteins of this pathway that are tumor suppressors can mutate and act as oncogenes. Therefore, inhibition of these signaling pathways by natural agents may be a strategy to target cancer cell progression inhibition, chemotherapeutic resistance to cancer cells as well as inhibition of self renewal property of cancer stem cells. While there are some reported inhibitors of Wnt and Notch pathways, there exists a significant unmet medical need to identify new, more efficient inhibitors.

The majorities of cancer drugs are small molecules designed to bind, interact and modulate the biological activity of the receptors. Computer aided drug designing (CADD) is being exploited to identify hits, pick leads and optimize drug leads by studying their physicochemical, pharmaceutical and ADME $\backslash \mathrm{T}$ (absorption, distribution, metabolism, excretion and toxicity) properties. The objective of CADD is to augment the set of molecules with prudent active, drug-like properties and abolish compounds with undesirable properties such as inactive, reactive, toxic, poor ADME/T. The ADEM/T property of compound gives some important in silico prediction of the lead compound that includes bio availability, blood brain barrier crossing and inhibition of $\mathrm{K}$ ion channels etc. It is broadly accepted that drug discovery and development are risky, costly, time and resource-consuming processes [3]. In silico screening approach is the leading technique for preliminary identification of natural products as inhibitors of targets protein and predicting their biological binding mode. In other words in silico modeling is used considerably to minimize time and resource requirements of chemical synthesis and biological in vitro and in vivo testing. While there are some reported inhibitors of Wnt and Notch pathways, there exists a significant unmet medical need to identify new, more efficient inhibitors of natural origin. These novel inhibitors may inhibit the cancer cell growth as well as drug resistance and self renewal ability of cancer stem-like cells. Furthermore, the Identified novel inhibitors may use as further anticancer leads for in vivo and clinical trial studies.

\section{References}

1. Phuah NH, Nagoor NH (2014) Regulation of MicroRNAs by Natural Agents: New Strategies in Cancer Therapies. BioMed Res Int 12014: 804510.

2. Yuan X, Wu H, Xu H, Xiong H, Chu Q, et al. (2015) Notch Signaling: An Emerging Therapeutic Target for Cancer Treatment. Cancer Lett 369(1): 20-27.

3. Isabella A Guedes, Camila S de Magalhães, Laurent E Dardenne (2014) Receptor-ligand molecular docking. Biophysical Reviews 6(1): 75-87. 